\title{
Reactions of $\mathrm{MoCl}_{5}$ and $\mathrm{MoO}_{2} \mathrm{Cl}_{2}$ with 4-Phenylimidazole-2-thiol and 2-Thiazoline-2-thiol
}

\author{
DEEPIKA RANI ${ }^{1}$, GURSHARAN SINGH ${ }^{2 *}$ and SEEMA SHARMA ${ }^{3}$
}

${ }^{1}$ Research Scholar Registered with Punjab Technical University, Kapurthala, India.

2Department of Applied Chemistry, Giani Zail Singh Campus College of Engineering and Technology, MRSPTU, Dabwali Road, Bathinda-151001, India.

3Department of Chemistry, Maharaja Ranjit Singh Punjab Technical University', Dabwali Road, Bathinda-151001, India.

*Correponding author E-mail: gursharans82@gmail.com

http://dx.doi.org/10.13005/ojc/360611

(Received: October 15, 2020; Accepted: November 17, 2020)

\section{ABSTRACT}

Reactions of $\mathrm{MoCl}_{5} / \mathrm{MoO}_{2} \mathrm{Cl}_{2}$ with 4-phenylimidazole-2-thiol/2-thiazoline-2-thiol in $\mathrm{CH}_{3} \mathrm{CN}$ solvent in 1:1/1:2 molar ratios have been carried out at room temperature. Products obtained $\mathrm{MoCl}_{3}\left(\mathrm{C}_{9} \mathrm{H}_{7} \mathrm{~N}_{2} \mathrm{~S}\right)\left(\mathrm{CH}_{3} \mathrm{CN}\right)$, [1]; $\mathrm{MoCl}_{2}\left(\mathrm{C}_{9} \mathrm{H}_{7} \mathrm{~N}_{2} \mathrm{~S}\right)\left(\mathrm{CH}_{3} \mathrm{CN}\right)$, [2]; $\mathrm{Mo}_{2} \mathrm{OCl}_{4}\left(\mathrm{C}_{9} \mathrm{H}_{8} \mathrm{~N}_{2} \mathrm{~S}\right)_{2}$, [3] and $\mathrm{Mo}_{4} \mathrm{O}_{2} \mathrm{Cl}_{12}\left(\mathrm{C}_{9} \mathrm{H}_{7} \mathrm{~N}_{2} \mathrm{~S}\right)_{4}$, [4]; $\mathrm{MoO}_{2} \mathrm{Cl}_{3}\left(\mathrm{C}_{3} \mathrm{H}_{5} \mathrm{NS}_{2}\right)_{2}$, [5] and $\mathrm{Mo}_{2} \mathrm{O}_{4} \mathrm{Cl}_{3}\left(\mathrm{C}_{3} \mathrm{H}_{5} \mathrm{NS}_{2}\right)_{2}$, [6] have been analyzed and characterized by elemental analysis, FTIR, ${ }^{1} \mathrm{H}$ NMR and LC-MS techniques. Compounds being moisture and air sensitive, these have been prepared in inert atmosphere using vacuum line and liquid nitrogen cooled traps. Fragments obtained in LC-MS spectra support the formulae derived.

Keywords: $\mathrm{MoCl}_{5}, \mathrm{MoO}_{2} \mathrm{Cl}_{2}$, 4-phenylimidazole-2-thiol, 2-thiazoline-2-thiol, FTIR, ${ }^{1} \mathrm{H}$ NMR and LC-MS, Fragments.

INTRODUCTION

Reactions of $\mathrm{MoCl}_{5}$ and $\mathrm{MoO}_{2} \mathrm{Cl}_{2}$ with various ligands have been reported in the literature. Earlier, $\mathrm{MoCl}_{5}$ reactions with 1,4-diaminobutane, potassium phthalimide, pyrazole, 2-mercaptopyridine$\mathrm{N}$-oxide sodium, imidazole, 2-methylpyridine, 3-methylpyridine, 4-methtylpyridine, succinimide, 2-thiazoline-2-thiol have been reported ${ }^{1,3-4,6-7}$ by the author. Reactions of $\mathrm{MoO}_{2} \mathrm{Cl}_{2}$ with 1,3diaminopropane, 1,4-diaminobutane, 1,3-propandiol, imidazole, pyrazole, acetamide, succinimide, potassium phthalimide, 2-thiazoline-2-thiol have also been reported ${ }^{1-3,6-7}$ by the author.

In the current paper, reactions of 4-phenylimidazole-2-thiol/2-thiazoline-2-thiol with $\mathrm{MoCl}_{5} / \mathrm{MoO}_{2} \mathrm{Cl}_{2}$ in $\mathrm{CH}_{3} \mathrm{CN}$ solvent at room temperature have been carried out to study addition, substitution, reduction, rearrangement or polymerization processes occurring.

${ }^{1}$ HNMR, FTIR of the compounds synthesized have been studied to determine the bonding of the ligands to Mo. Fragmentation pattern

This is an Open Access article licensed under a Creative Commons license: Attribution 4.0 International (CC- BY). Published by Oriental Scientific Publishing Company @ 2018

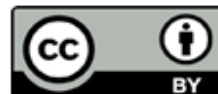


of the compounds observed in LC-MS mass spectra support molecular formulae derived.

\section{AIM of Investigation}

Heterocyclic thioamides have $\mathrm{N}$ and S-donor ligands which are known to form different types of coordination compounds. They are used in analysis and corrosion control. They are biologically active. $\mathrm{N}$-methylimidazoline-2-thione and other thioamides are used as anti-thyroidal agents ${ }^{8}$. Heterocyclic compounds having imidazothiazole structural unit are biologically active ${ }^{9}$. They have the ability to inhibit or activate many enzymes and receptors ${ }^{10-13}$. They act as antitumor ${ }^{14-19}$, antimicrobial ${ }^{20-21}$, antidiabetic ${ }^{22}$, diuretic ${ }^{23}$, antihelmintic ${ }^{24}$ and fungicidal ${ }^{25}$ compounds.

Molybdenum compounds containing 2-thiazoline-2-thiol have been prepared by the author2, 5-6. Metal complexes with sulphur containing ligands have many biochemical applications ${ }^{26-28}$.

\section{MATERIALS AND METHODS}

$\mathrm{MoO}_{2} \mathrm{Cl}_{2}, \mathrm{MoCl}_{5}$ and 2-thiazoline-2-thiol were purchased from Sigma-Aldrich, USA and used as such.

Mo and $\mathrm{Cl}$ have been estimated gravimetrically by oxinate method ${ }^{29}$ and silver chloride method ${ }^{29}$, respectively. Other elements were analysed using Thermo Finnigan Elemental Analyzer. Perkin-Elmer 400 FTIR Spectrometer, in $\mathrm{KBr}$ disks was used to record spectra in the range $4000-400$ $\mathrm{cm}^{-1}$. Brucker Avance-Il 400 NMR in DMSO- $\mathrm{d}_{6}$ was used for obtaining ${ }^{1} \mathrm{H}-\mathrm{NMR}$ spectra. WATERS, Q-TOF Micromass LC-MS (UK) was used for LC-MS spectra in the range $0-1100 \mathrm{~m} / \mathrm{z}$. These facilities were availed at SAIF/CIL, Panjab University, Chandigarh (India).

\section{Synthesis of compounds [1]-[6]}

Pressure stabilized dropping funnel having teflon stop-cock was connected to a $100 \mathrm{~mL}$ flask. For stirring, a magnetic bead was placed in the flask. Apparatus was connected to vacuum line and dried by heating. On cooling, apparatus was flushed with oxygen purged dry nitrogen gas. Known amount of $\mathrm{MoO}_{2} \mathrm{Cl}_{2}$ or $\mathrm{MoCl}_{5}$ was placed in the flask along with dry $\mathrm{CH}_{3} \mathrm{CN}$ solvent. 4-phethylimidazole-2-thiol or 2-thiazoline-2-thiol was placed in equimolar or 1:2 molar amount along with $\mathrm{CH}_{3} \mathrm{CN}$ solvent in dropping funnel. Solution from the dropping funnel was added to $\mathrm{MoO}_{2} \mathrm{Cl}_{2}$ or $\mathrm{MoCl}_{5}$ placed in bottom flask at room temperature with continuous stirring. Compounds thus prepared were filtered under reduced pressure through filtration unit. Compounds prepared have sensitivity to air and moisture. On exposure to air and moisture, their colour changes to blue. All procedures and work up have been done in vacuum line using oxygen purged under dry nitrogen gas. Moisture and oxygen were further removed using liquid nitrogen cooled traps.

Rearrangement and disproportionation have occurred during the reactions. F/R means filtrate/residue yielding the product.

$$
\begin{aligned}
& \mathrm{MoCl}_{5}+2 \mathrm{C}_{9} \mathrm{H}_{8} \mathrm{~N}_{2} \mathrm{~S} \stackrel{\mathrm{CH}_{3} \mathrm{CN}}{\longrightarrow: 2} \mathrm{MoCl}_{3}\left(\mathrm{C}_{9} \mathrm{H}_{7} \mathrm{~N}_{2} \mathrm{~S}\right)\left(\mathrm{CH}_{3} \mathrm{CN}\right),[1] \\
& \text { 4- Phenylimidazole - } 2 \text { - thiol Green (F) } \\
& \mathrm{MoCl}_{5}+\mathrm{C}_{9} \mathrm{H}_{8} \mathrm{~N}_{2} \mathrm{~S} \stackrel{\mathrm{CH}_{3} \mathrm{CN}}{\longrightarrow: 1} \mathrm{MoCl}_{2}\left(\mathrm{C}_{9} \mathrm{H}_{8} \mathrm{~N}_{2} \mathrm{~S}\right)\left(\mathrm{CH}_{3} \mathrm{CN}\right) \text {, [2] } \\
& 4 \text { - Phenylimidazole - } 2 \text { - thiol Light blue (R) } \\
& \mathrm{MoO}_{2} \mathrm{Cl}_{2}+\mathrm{C}_{9} \mathrm{H}_{8} \mathrm{~N}_{2} \mathrm{~S} \stackrel{\mathrm{CH}_{3} \mathrm{CN}}{\mathrm{i}: 1} \mathrm{Mo}_{2} \mathrm{OCl}_{4}\left(\mathrm{C}_{9} \mathrm{H}_{8} \mathrm{~N}_{2} \mathrm{~S}\right)_{2},[3] \\
& \text { 4- Phenylimidazole - } 2 \text { - thiol Black (R) } \\
& \mathrm{MoO}_{2} \mathrm{Cl}_{2}+\mathrm{C}_{9} \mathrm{H}_{8} \mathrm{~N}_{2} \mathrm{~S} \stackrel{\mathrm{CH}_{3} \mathrm{CN}}{\longrightarrow} \mathrm{MoO}_{2} \mathrm{Cl}_{3}\left(\mathrm{C}_{9} \mathrm{H}_{8} \mathrm{~N}_{2} \mathrm{~S}\right) \text {, [4] } \\
& 4 \text { - Phenylimidazole - } 2 \text { - thiol Greyish blue (F) } \\
& \mathrm{MoO}_{2} \mathrm{Cl}_{2}+\mathrm{C}_{3} \mathrm{H}_{5} \mathrm{NS}_{2} \stackrel{\mathrm{CH}_{3} \mathrm{CN}}{\longrightarrow: 2} \mathrm{MoO}_{2} \mathrm{Cl}_{3}\left(\mathrm{C}_{3} \mathrm{H}_{5} \mathrm{NS}_{2}\right)_{2},[5] \\
& \text { 2-Thiazoline - 2 - thiol Dark brown (R) } \\
& \mathrm{MoO}_{2} \mathrm{Cl}_{2}+\mathrm{C}_{3} \mathrm{H}_{5} \mathrm{NS}_{2} \stackrel{\mathrm{CH}_{3} \mathrm{CN}}{\longrightarrow: 1} \mathrm{Mo}_{2} \mathrm{O}_{4} \mathrm{Cl}_{3}\left(\mathrm{C}_{3} \mathrm{H}_{5} \mathrm{NS}_{2}\right)_{2},[6] \\
& \text { 2- Thiazoline - 2 - thiol Greenish blue (F) }
\end{aligned}
$$

\begin{tabular}{|c|c|c|c|c|c|c|}
\hline \multirow{2}{*}{$\frac{\text { Compounds }}{\text { (Colour/Formula Mass) }}$} & \multicolumn{6}{|c|}{$\%$ Observed (Theoretical) } \\
\hline & Mo & $\mathrm{Cl}$ & $\mathrm{C}$ & $\mathrm{H}$ & $\mathrm{N}$ & S \\
\hline $\begin{array}{c}\mathrm{MoCl}_{3}\left(\mathrm{C}_{9} \mathrm{H}_{7} \mathrm{~N}_{2} \mathrm{~S}\right)\left(\mathrm{CH}_{3} \mathrm{CN}\right),[1] \\
(\mathrm{Green} / 418.5)\end{array}$ & $\begin{array}{c}23.76 \\
(22.93)\end{array}$ & $\begin{array}{c}24.76 \\
(25.44)\end{array}$ & $\begin{array}{c}30.22 \\
(31.54)\end{array}$ & $\begin{array}{l}3.01 \\
(2.38)\end{array}$ & $\begin{array}{c}9.41 \\
(10.03)\end{array}$ & $\begin{array}{l}7.31 \\
(7.64)\end{array}$ \\
\hline $\begin{array}{c}\mathrm{MoCl}_{2}\left(\mathrm{C}_{9} \mathrm{H}_{7} \mathrm{~N}_{2} \mathrm{~S}\right)\left(\mathrm{CH}_{3} \mathrm{CN}\right),[2] \\
\text { (Light blue/383.0) }\end{array}$ & $\begin{array}{l}25.35 \\
(25.06)\end{array}$ & $\begin{array}{l}17.73 \\
(18.53)\end{array}$ & $\begin{array}{c}33.66 \\
(34.46)\end{array}$ & $\begin{array}{l}2.91 \\
(2.61)\end{array}$ & $\begin{array}{c}10.08 \\
(10.96)\end{array}$ & $\begin{array}{c}9.21 \\
(8.35)\end{array}$ \\
\hline $\begin{array}{c}\mathrm{Mo}_{2} \mathrm{OCl}_{4}\left(\mathrm{C}_{9} \mathrm{H}_{8} \mathrm{~N}_{2} \mathrm{~S}\right)_{2},[3] \\
(\text { Black/702.0) }\end{array}$ & $\begin{array}{l}28.03 \\
(27.35)\end{array}$ & $\begin{array}{c}21.00 \\
(20.22)\end{array}$ & $\begin{array}{c}29.99 \\
(30.76)\end{array}$ & $\begin{array}{c}2.61 \\
(2.27)\end{array}$ & $\begin{array}{l}7.32 \\
(7.97)\end{array}$ & $\begin{array}{l}8.35 \\
(9.11)\end{array}$ \\
\hline $\begin{array}{c}\mathrm{Mo}_{4} \mathrm{O}_{2} \mathrm{Cl}_{12}\left(\mathrm{C}_{9} \mathrm{H}_{7} \mathrm{~N}_{2} \mathrm{~S}\right)_{4},[4] \\
\text { (Greyish blue/1546.0) }\end{array}$ & $\begin{array}{c}25.63 \\
(24.83)\end{array}$ & $\begin{array}{l}28.46 \\
(27.55)\end{array}$ & $\begin{array}{c}27.07 \\
(27.94)\end{array}$ & $\begin{array}{c}2.77 \\
(1.81)\end{array}$ & $\begin{array}{c}6.86 \\
(7.24)\end{array}$ & $\begin{array}{c}7.50 \\
(8.27)\end{array}$ \\
\hline $\begin{array}{c}\mathrm{MoO}_{2} \mathrm{Cl}_{3}\left(\mathrm{C}_{3} \mathrm{H}_{5} \mathrm{NS}_{2}\right)_{2},[5] \\
\text { (Dark brown/472.5) }\end{array}$ & $\begin{array}{l}21.16 \\
(20.31)\end{array}$ & $\begin{array}{l}22.58 \\
(22.53)\end{array}$ & $\begin{array}{c}14.56 \\
(15.23)\end{array}$ & $\begin{array}{l}2.08 \\
(2.11)\end{array}$ & $\begin{array}{c}5.62 \\
(5.92)\end{array}$ & $\begin{array}{c}26.99 \\
(27.08)\end{array}$ \\
\hline $\mathrm{Mo}_{2} \mathrm{O}_{4} \mathrm{Cl}_{3}\left(\mathrm{C}_{3} \mathrm{H}_{5} \mathrm{NS}_{2}\right)_{2},[6]$ & 32.56 & 17.40 & 11.32 & 2.28 & 4.60 & 20.47 \\
\hline (Greenish blue/600.5) & (31.97) & $(17.73)$ & (11.99) & $(1.67)$ & $(4.66)$ & (21.31) \\
\hline
\end{tabular}

Analytical studies

Table 1: (Elemental Analysis) 


\section{FTIR Spectra}

There is an increase of $145,149,150 \& 148$ $\mathrm{cm}^{-1}$ in $\mathrm{v}(\mathrm{N}-\mathrm{H})$ of 4-phenylimidazole-2-thio ${ }^{30-32}$ in [1], [2], [3] \& [4], respectively (Table 2) showing the presence of $\mathrm{N}-\mathrm{H}$ group. There is no absorption in the region 2550-2600 $\mathrm{cm}^{-1}$ of [1], [2], [3] \& [4] corresponding to $\mathrm{v}(\mathrm{S}-\mathrm{H})$, conveying absence of $\mathrm{S}-\mathrm{H}$ group. $\mathrm{v}(\mathrm{C}=\mathrm{S})$ are absent in these compounds. $v(C=S)$ is observed at a lower wave number than $\mathrm{v}(\mathrm{C}=\mathrm{O})$, because $\mathrm{C}=\mathrm{S}$ bond is weaker and less polar than $\mathrm{C}=\mathrm{O}$ bond. $\mathrm{C}=\mathrm{S}$ absorptions are less intense than those of $\mathrm{C}=\mathrm{O}$ group. Absorption at 762, 761, 762 \& $762 \mathrm{~cm}^{-1}$ in [1], [2], [3] \& [4], respectively is associated with $\mathrm{u}(\mathrm{C}-\mathrm{S})$. Presence of $\mathrm{v}(\mathrm{C}-\mathrm{S})$ may be due to formation of Mo-S bond.

Table 2: (Infrared Absorptions in $\mathrm{cm}^{-1}$ )

\begin{tabular}{|c|c|c|c|c|c|}
\hline Assignment & $\begin{array}{c}\mathrm{C}_{9} \mathrm{H}_{7} \mathrm{~N}_{2} \mathrm{~S} \\
\text { (4-Phenylimidazole } \\
\text {-2-thiol) }\end{array}$ & [1] & [2] & [3] & [4] \\
\hline $\begin{array}{l}\mathrm{v}(\mathrm{N}-\mathrm{H}) \\
\mathrm{v}(\mathrm{S}-\mathrm{H})\end{array}$ & $3129,3248 \mathrm{~s}$ & $\begin{array}{c}3393.7 \text { v. s. } \\
--\end{array}$ & $\begin{array}{c}3396.1 \text { v. s. } \\
--\end{array}$ & $\begin{array}{c}3396.6 \text { v. s. } \\
---\end{array}$ & $\begin{array}{c}3397.7 \text { v. s. } \\
---\end{array}$ \\
\hline$v(C=C), v(C=N)$ & $1558,1500,1465$ & $1623.2 \mathrm{~s}, 1593.2 \mathrm{sh}, 1457.2 \mathrm{~m}$ & $\begin{array}{l}1621.3 \mathrm{~s} \\
1459.1 \mathrm{~m}\end{array}$ & $\begin{array}{c}1619.8 \mathrm{~s}, 1597.3 \mathrm{sh} \\
1456.9 \mathrm{w}\end{array}$ & $\begin{array}{c}1621.7 \mathrm{~s}, 1598.2 \mathrm{sh}, \\
1456.6 \mathrm{w}\end{array}$ \\
\hline$v(C=S)$ & 1261,1109 & ---- & ---- & --- & --- \\
\hline$v(C-S)$ & 780 & $762.4 \mathrm{~s}$ & $761.7 \mathrm{~s}$ & $762.9 \mathrm{~s}$ & $762.2 \mathrm{~s}$ \\
\hline$v(\text { Mo-S })^{33}$ & 421 & --- & --- & --- & --- \\
\hline$v_{\text {terminal }}(\mathrm{Mo}=0)^{34-36}$ & --- & --- & --- & $981.3 \mathrm{~s}$ & $981.7 \mathrm{~s}$ \\
\hline
\end{tabular}

$\mathrm{v}(\mathrm{Mo}=\mathrm{O})$ occurs $^{33}$ in the range $990-1010 \mathrm{~cm}^{-1}$. Absorption at $981 \& 981 \mathrm{~cm}^{-1}$ in [3] \& [4], respectively corresponds to terminal $\mathrm{v}(\mathrm{Mo}=\mathrm{O})^{34-36}$

Thiol-thione tautomerism is typical of imidazole-2-thiones ${ }^{32}$. There is a decrease in $v(\mathrm{Mo}=\mathrm{O})$ which shows $\mathrm{S} \rightarrow$ Mo coordination ${ }^{37}$ of 4-phenylimidazole-2-thiol in a direction trans to $\mathrm{Mo}=\mathrm{O}$ bond. This shows that 4-phenylimidazole-2thione reacted in a thiol form.

There is an increase of 285 \& zero $\mathrm{cm}^{-1}$ in $v(\mathrm{~N}-\mathrm{H})$ of 2-thiazoline-2-thiol in [5] \& [6], respectively (Table 3). The spectrum does not show absorption around $2710 \mathrm{~cm}^{-1}$ conveying $\mathrm{S}-\mathrm{H}$ group is absent in [5] \& [6]. Presence of $v(C=S)$ at $1308 \& 1309 \mathrm{~cm}^{-1}$ in [5] \& [6], respectively and further, absence of
$v(\mathrm{C}=\mathrm{N})$ indicate that $\mathrm{S}-\mathrm{H}$ bond is missing in these compounds. Ligand is attached in thio-keto form in them. Bonding of ligand seems to be through $\mathrm{S} \rightarrow \mathrm{Mo}$ coordinate bond.

Absorption at $963 \mathrm{~cm}^{-1}$ in [5] corresponds to terminal $v(\mathrm{Mo}=\mathrm{O})^{34-36}$. There is a decrease in $v(\mathrm{Mo}=\mathrm{O})$ which shows coordination ${ }^{37}$ of 2-thiazoline2-thiol to Mo through $S$ atom in a direction trans to $\mathrm{Mo}=\mathrm{O}$ bond.

Bands at $983 \& 960 \mathrm{~cm}^{-1}$ in [6] indicate the presence of cis- $\mathrm{MoO}_{2}{ }^{2+}$ core ${ }^{38}$.

Table 3: (Infrared Absorptions in $\mathrm{cm}^{-1}$ )

\begin{tabular}{|c|c|c|c|}
\hline Assignment & $\mathrm{C}_{3} \mathrm{H}_{5} \mathrm{NS}_{2}$ 2-Thiazoline-2-thiol ${ }^{30-32}$ & {$[5]$} & {$[6]$} \\
\hline$v(\mathrm{~N}-\mathrm{H})$ asym. & 3145 & 3429.7 vs & $3145.0 \mathrm{~s}$ \\
\hline$v(\mathrm{~S}-\mathrm{H})$ & 2709 & ---- & --- \\
\hline$v(\mathrm{C}=\mathrm{N})$ & $1518 \mathrm{~s}$ & ---- & ---- \\
\hline$v(C=S)$ & $1300 \mathrm{~m}$ & $1308.4 \mathrm{~s}$ & $1309.8 \mathrm{sh}$ \\
\hline$v_{\text {ring }}(C-N)$ & $1260 \mathrm{sh}$ & $1254.8 v w$ & $1256.4 \mathrm{w}$ \\
\hline$v_{\text {sym. }}(C-N)$ & 1218 & $1202.5 \mathrm{~m}$ & $1208.6 \mathrm{~m}$ \\
\hline$v(\mathrm{Mo}-\mathrm{N})$ & --- & $458.6 \mathrm{sh}$ & $460.1 \mathrm{sh}$ \\
\hline$v($ Mo-S) & 421 & --- & --- \\
\hline$v_{\text {terminal }}(\mathrm{Mo}=0) 34-36$ & --- & $963.0 \mathrm{~s}$ & --- \\
\hline cis- $\mathrm{MoO}_{2}{ }^{2+} \operatorname{core}^{38} \mathrm{v}(\mathrm{Mo}=\mathrm{O})$ & --- & -- & $984.0 \mathrm{sh}, 959.0 \mathrm{vs}$ \\
\hline
\end{tabular}

\section{${ }^{1} \mathrm{H}$ NMR Spectra}

4-Phenylimidazole-2-thiol ${ }^{39,40}$ has $\mathrm{N}-\mathrm{H}$ peak at 13.00 ppm. Since $-\mathrm{OH},-\mathrm{NH}_{2},-\mathrm{SH}$ are labile protons and spectrum is taken in some solvent, so they have no characteristic chemical shift. No $\mathrm{N}-\mathrm{H}$ peak has been observed in [1], [2], [3] \& [4] (Table 4). 
4-Phenylimidazole-2-thiol has S-H peak at $12.15 \mathrm{ppm}$. No S-H peak has been observed in [1], [2], [3] \& [4]. This indicates the absence of S-H group in [1], [2], [3] \& [4]. There has been upfield shift of $\mathrm{H}-5$ in all the four compounds. All the ring protons of phenyl group have also shown up field shift in all the four compounds. Presence of $\mathrm{CH}_{3} \mathrm{CN}$ in [1] \& [2] has been inferred by the absorptions at $2.05 \& 1.98 \mathrm{ppm}$, respectively.

Table 4: ( ${ }^{1} \mathrm{H}$ NMR absorptions in ppm)

\begin{tabular}{|c|c|c|c|c|c|}
\hline Assignment & $\mathrm{C}_{9} \mathrm{H}_{7} \mathrm{~N}_{2} \mathrm{~S}$ (4-Phenylimidazole-2-thiol) & {$[1]$} & {$[2]$} & {$[3]$} & {$[4]$} \\
\hline $\mathrm{N}-\mathrm{H}$ & 13.00 & --- & -- & --- & --- \\
\hline $\mathrm{S}-\mathrm{H}$ & 12.15 & --- & --- & --- & --- \\
\hline $\mathrm{H}-5$ & 7.51 & 7.47 & 7.41 & 7.33 & 7.39 \\
\hline Phenyl H-2, H-6 & 8.13 & 7.90 & 7.86 & 7.76 & 7.77 \\
\hline Phenyl H-3, H-5 & 7.51 & 7.47 & 7.41 & 7.33 & 7.45 \\
\hline Phenyl H-4 & 7.41 & --- & --- & --- & --- \\
\hline $\mathrm{CH}_{3} \mathrm{CN}$ & --- & 2.05 & 1.98 & --- & --- \\
\hline
\end{tabular}

$\mathrm{N}-\mathrm{H}$ absorptions have not been observed in [5] \& [6] (Table 5). Other protons have shown downfield shift in them showing $\mathrm{S} \rightarrow$ Mo coordination.

\section{LC-MS MASS Spectra ${ }^{42}$}

Fragmentation pattern obtained below has been used to derive the formulae (Table 6,7 ). $\mathrm{m} / \mathrm{z}$ values have been given below of the fragments.

Table 5: ( ${ }^{1} \mathrm{H}$ NMR absorptions in ppm)

\begin{tabular}{ccccc}
\hline Assignment & $\mathrm{C}_{3} \mathrm{H}_{5} \mathrm{NS}_{2}$ & 2-Thiazoline-2-thiol41 & {$[5]$} & {$[6]$} \\
\hline $\mathrm{CH}_{2}$ attached to $\mathrm{N}$ & $3.33(2 \mathrm{H})$ & 3.27 & 3.41 \\
$\mathrm{CH}_{2}$ attached to $\mathrm{S}$ & $3.56(2 \mathrm{H})$ & 3.88 & 3.83 \\
$\mathrm{~N}-\mathrm{H}$ & $7.43(1 \mathrm{H})$ & --- & --- \\
$\mathrm{S}-\mathrm{H}$ & & --- & --- \\
\hline
\end{tabular}

Table 6: (Fragmentation)

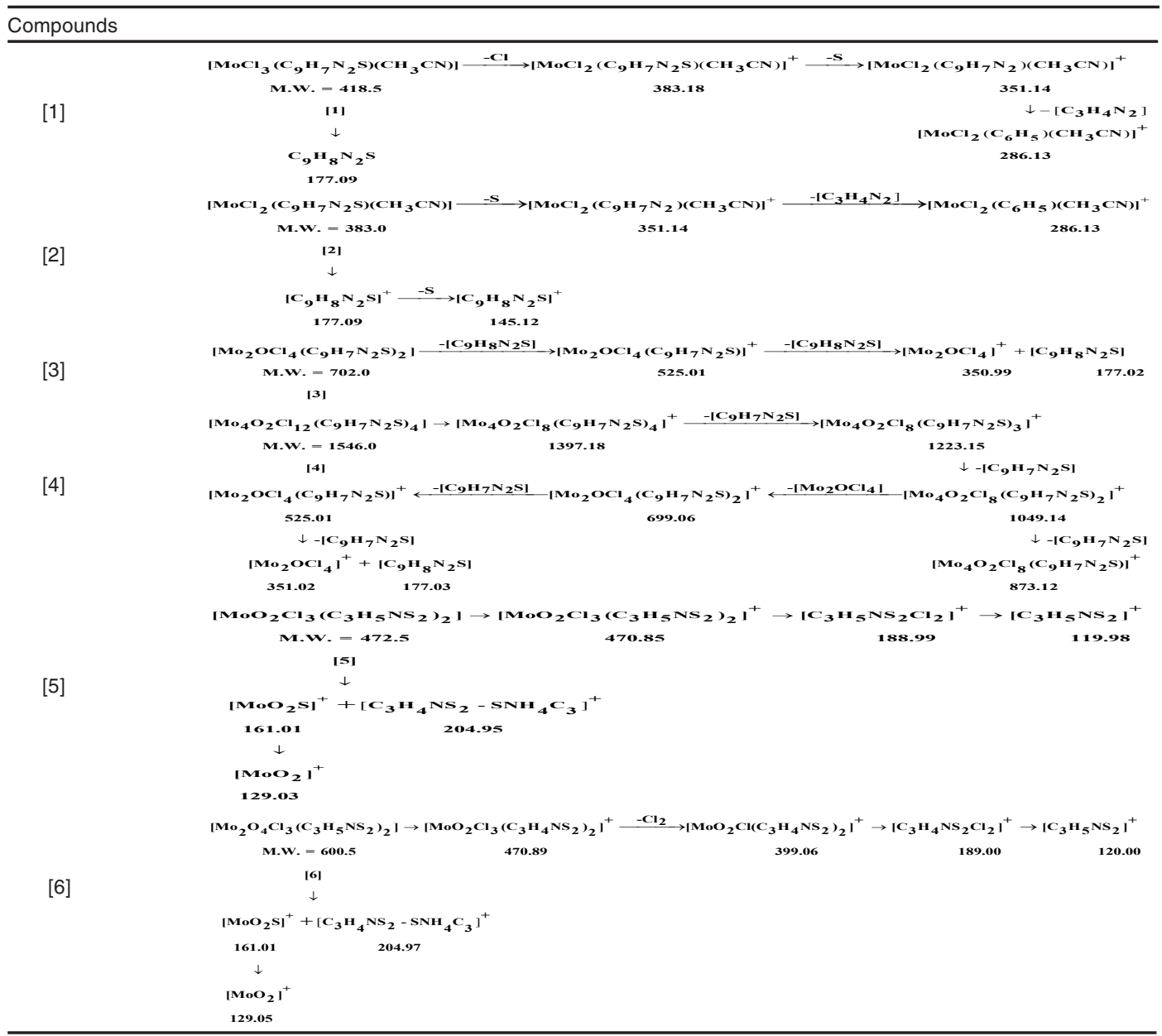


Table: 7

\begin{tabular}{|c|c|c|c|c|}
\hline Compounds & Fragment & Calculated ${ }^{35} \mathrm{~m} / \mathrm{z}$ & Recorded $\mathrm{m} / \mathrm{z}$ & Rel. abundance \\
\hline \multirow[t]{5}{*}[1]{} & {$\left[\mathrm{C}_{9} \mathrm{H}_{8} \mathrm{~N}_{2} \mathrm{~S}\right]^{+}$} & 176.04 & 177.09 & $40 \%$ \\
\hline & {$\left[\mathrm{MoCl}_{2}\left(\mathrm{C}_{9} \mathrm{H}_{7} \mathrm{~N}_{2} \mathrm{~S}\right)\left(\mathrm{CH}_{3} \mathrm{CN}\right)\right]^{+}$} & 384.91 & 383.18 & $5 \%$ \\
\hline & {$\left[\mathrm{MoCl}_{2}\left(\mathrm{C}_{6} \mathrm{H}_{5}\right)\left(\mathrm{CH}_{3} \mathrm{CN}\right)\right]^{+}$} & 285.90 & 286.13 & $30 \%$ \\
\hline & {$\left[\mathrm{MoCl}\left(\mathrm{C}_{6} \mathrm{H}_{5}\right)\left(\mathrm{CH}_{3} \mathrm{CN}\right)\right]^{+}$} & 250.93 & 252.14 & $10 \%$ \\
\hline & {$\left[\mathrm{MoCl}_{2}\left(\mathrm{C}_{9} \mathrm{H}_{8} \mathrm{~N}_{2}\right)\left(\mathrm{CH}_{3} \mathrm{CN}\right)\right]^{+}$} & 352.93 & 351.14 & $100 \%$ \\
\hline \multirow[t]{5}{*}[2]{} & {$\left[\mathrm{MoCl}_{2}\left(\mathrm{C}_{9} \mathrm{H}_{8} \mathrm{~N}_{2} \mathrm{~S}\right)\left(\mathrm{CH}_{3} \mathrm{CN}\right)\right]^{+}$} & 384.91 & 383.18 & $6 \%$ \\
\hline & {$\left[\mathrm{C}_{9} \mathrm{H}_{8} \mathrm{~N}_{2} \mathrm{~S}\right]^{+}$} & 176.04 & 177.09 & $41 \%$ \\
\hline & {$\left[\mathrm{C}_{9} \mathrm{H}_{8} \mathrm{~N}_{2}\right]^{+}$} & 144.06 & 145.12 & $7 \%$ \\
\hline & {$\left[\mathrm{MoCl}_{2}\left(\mathrm{C}_{9} \mathrm{H}_{8} \mathrm{~N}_{2}\right)\left(\mathrm{CH}_{3} \mathrm{CN}\right)\right]^{+}$} & 352.93 & 351.14 & $100 \%$ \\
\hline & {$\left[\mathrm{MoCl}_{2}\left(\mathrm{C}_{6} \mathrm{H}_{5}\right)\left(\mathrm{CH}_{3} \mathrm{CN}\right)\right]^{+}$} & 285.90 & 286.13 & $7 \%$ \\
\hline \multirow[t]{4}{*}{ [3] } & {$\left[\mathrm{C}_{9} \mathrm{H}_{8} \mathrm{~N}_{2} \mathrm{~S}\right]^{+}$} & 176.04 & 177.02 & $28 \%$ \\
\hline & {$\left[\mathrm{Mo}_{2} \mathrm{OCl}_{4}\right]^{+}$} & 351.68 & 350.99 & $100 \%$ \\
\hline & {$\left[\mathrm{Mo}_{2} \mathrm{OCl}_{4}\left(\mathrm{C}_{9} \mathrm{H}_{7} \mathrm{~N}_{2} \mathrm{~S}\right)\right]$} & 526.71 & 525.01 & $40 \%$ \\
\hline & {$\left[\mathrm{Mo}_{2} \mathrm{OCl}_{4}\left(\mathrm{C}_{9} \mathrm{H}_{7} \mathrm{~N}_{2} \mathrm{~S}\right)_{2}\right]^{+}$} & 701.74 & 699.00 & $2 \%$ \\
\hline \multirow[t]{8}{*}[4]{} & {$\left[\mathrm{C}_{9} \mathrm{H}_{8} \mathrm{~N}_{2} \mathrm{~S}\right]^{+}$} & 176.04 & 177.03 & $28 \%$ \\
\hline & {$\left[\mathrm{Mo}_{2} \mathrm{OCl}_{4}\right]^{+}$} & 351.68 & 351.02 & $78 \%$ \\
\hline & {$\left[\mathrm{Mo}_{2} \mathrm{OCl}_{4}\left(\mathrm{C}_{9} \mathrm{H}_{7} \mathrm{~N}_{2} \mathrm{~S}\right)\right]$} & 526.71 & 525.01 & $100 \%$ \\
\hline & {$\left[\mathrm{Mo}_{2} \mathrm{OCl}_{4}\left(\mathrm{C}_{9} \mathrm{H}_{7} \mathrm{~N}_{2} \mathrm{~S}\right)_{2}\right]^{+}$} & 701.74 & 699.06 & $40 \%$ \\
\hline & {$\left[\mathrm{Mo}_{4} \mathrm{O}_{2} \mathrm{Cl}_{8}\left(\mathrm{C}_{9} \mathrm{H}_{7} \mathrm{~N}_{2} \mathrm{~S}\right)\right]^{+}$} & 878.39 & 873.12 & $3 \%$ \\
\hline & {$\left[\mathrm{Mo}_{4} \mathrm{O}_{2} \mathrm{Cl}_{8}\left(\mathrm{C}_{9} \mathrm{H}_{7} \mathrm{~N}_{2} \mathrm{~S}\right)_{2}\right]^{+}$} & 1053.42 & 1049.14 & $<1 \%$ \\
\hline & {$\left[\mathrm{Mo}_{4} \mathrm{O}_{2} \mathrm{Cl}_{8}\left(\mathrm{C}_{9} \mathrm{H}_{7} \mathrm{~N}_{2} \mathrm{~S}\right)_{3}\right]^{+}$} & 1228.46 & 1223.15 & $<1 \%$ \\
\hline & {$\left[\mathrm{Mo}_{4} \mathrm{O}_{2} \mathrm{Cl}_{8}\left(\mathrm{C}_{9} \mathrm{H}_{7} \mathrm{~N}_{2} \mathrm{~S}\right)_{4}\right]^{+}$} & 1403.48 & 1397.18 & $<1 \%$ \\
\hline \multirow[t]{6}{*}[5]{} & {$\left[\mathrm{MoO}_{2} \mathrm{Cl}_{3}\left(\mathrm{C}_{3} \mathrm{H}_{4} \mathrm{NS}_{2}\right)_{2}\right]^{+}$} & 470.75 & 470.85 & $4 \%$ \\
\hline & {$\left[\mathrm{C}_{3} \mathrm{H}_{5} \mathrm{NS}_{2}\right]^{+}$} & 118.98 & 119.98 & $8 \%$ \\
\hline & {$\left[\mathrm{MoO}_{2}\right]^{+}$} & 129.89 & 129.03 & $28 \%$ \\
\hline & {$\left[\mathrm{MoO}_{2} \mathrm{~S}\right]^{+}$} & 161.86 & 161.01 & $8 \%$ \\
\hline & {$\left[\mathrm{C}_{3} \mathrm{H}_{4} \mathrm{NS}_{2} \mathrm{SNH}_{4} \mathrm{C}_{3}\right]^{+}$} & 203.98 & 204.95 & $100 \%$ \\
\hline & {$\left[\mathrm{C}_{3} \mathrm{H}_{5} \mathrm{NS}_{2} \mathrm{Cl}_{2}\right]^{+}$} & 188.92 & 188.99 & $44 \%$ \\
\hline \multirow[t]{7}{*}[6]{} & {$\left[\mathrm{MoO}_{2} \mathrm{Cl}_{3}\left(\mathrm{C}_{3} \mathrm{H}_{4} \mathrm{NS}_{2}\right)_{2}\right]^{+}$} & 470.75 & 470.85 & $2 \%$ \\
\hline & {$\left[\mathrm{C}_{3} \mathrm{H}_{5} \mathrm{NS}_{2}\right]^{+}$} & 118.98 & 120.00 & $1 \%$ \\
\hline & {$\left[\mathrm{MoO}_{2}\right]^{+}$} & 129.89 & 129.05 & $25 \%$ \\
\hline & {$\left[\mathrm{MoO}_{2} \mathrm{~S}\right]^{+}$} & 161.86 & 161.01 & $20 \%$ \\
\hline & {$\left[\mathrm{C}_{3} \mathrm{H}_{5} \mathrm{NS}_{2} \mathrm{Cl}_{2}\right]^{+}$} & 188.92 & 189.00 & $86 \%$ \\
\hline & {$\left[\mathrm{C}_{3} \mathrm{H}_{4} \mathrm{NS}_{2} \mathrm{SNH}_{4} \mathrm{C}_{3}\right]^{+}$} & 203.98 & 204.97 & $100 \%$ \\
\hline & {$\left[\mathrm{MoO}_{2} \mathrm{Cl}\left(\mathrm{C}_{3} \mathrm{H}_{4} \mathrm{NS}_{2}\right)_{2}\right]^{+}$} & 400.82 & 399.06 & $3 \%$ \\
\hline
\end{tabular}

\section{CONCLUSION}

There is no absorption in the region 2550$2600 \mathrm{~cm}^{-1}$ of [1], [2], [3] \& [4] corresponding to $\mathrm{v}(\mathrm{S}-\mathrm{H})$, showing absence of $\mathrm{S}-\mathrm{H}$ group. $\mathrm{v}(\mathrm{C}=\mathrm{S})$ stretchings are absent in [1], [2], [3] \& [4]. Absorptions at 762, $761,762 \& 762 \mathrm{~cm}^{-1}$ in [1], [2], [3] \& [4], respectively suggest presence of $\mathrm{u}(\mathrm{C}-\mathrm{S})$ of Mo-S bonds.

Presence of $v(C=S)$ at $1308 \& 1309 \mathrm{~cm}^{-1}$ in [5] \& [6], respectively and further, absence of $v(C=N)$ in [5] \& [6], respectively indicate that $\mathrm{S}-\mathrm{H}$ bond is missing. Bonding of ligand seems to be through $\mathrm{S} \rightarrow$ Mo coordinate bond.

No characteristic S-H chemical shift has been observed in all the six compounds, indicating that either $\mathrm{C}=\mathrm{S}$ is present or Mo-S is present in these compounds. Presence of $\mathrm{CH}_{3} \mathrm{CN}$ in [1] \& [2] has been inferred due to presence of ${ }^{1} \mathrm{H}$ NMR peak of $\mathrm{CH}_{3} \mathrm{CN}$ in them.

LC-MS spectra support the presence of particular ligands in these compounds and their proposed formulae.

\section{AACKNOWLEDGEMENT}

We acknowledge our thanks to SAIF/CIL, Panjab University, Chandigarh (India) for extending us the characterising facility for LC-MS, elemental analysis, $1 \mathrm{H}-\mathrm{NMR} \&$ FTIR.

\section{Conflict of interest}

It is declared that the authors have no conflict of interest. 


\section{REFERENCES}

1. Singh, G.; Mangla, V.; Goyal, M.; Singla, K.; Rani, D., American International Journal of Research in Science, Technology, Engineering \& Mathematics., 2014, 8(2), 131-136.

2. Singh, G.; Mangla, V.; Goyal, M.; Singla, K.; Rani, D., American International Journal of Research in Science, Technology, Engineering \& Mathematics., 2015, 9(1), 25-33.

3. Singh, G.; Mangla, V.; Goyal, M.; Singla, K.; Rani, D., American International Journal of Research in Science, Technology, Engineering \& Mathematics., 2015, 10(4), 299-308.

4. Singh, G.; Mangla, V.; Goyal, M.; Singla, K.; Rani, D.; Rakesh, K., American International Journal of Research in Science, Technology, Engineering \& Mathematics., 2015, 11(2), 158-166.

5. Singh, G.; Mangla, V.; Goyal, M.; Singla, K.; Rani, D., International Congress on Chemical, Biological and Environmental Sciences., May, Kyoto (Japan)., 2015, 7-9, 930-942.

6. Singh, G.; Mangla, V.; Goyal, M.; Singla, K.; Rani, D.; Kumar, R., American International Journal of Research in Science, Technology, Engineering \& Mathematics., 2016, 16(1), 56-64.

7. Singh, G.; Kumar, R., American International Journal of Research in Science, Technology, Engineering \& Mathematics., 2018, 22(1), 01-08.

8. Owczarzak, A. M.; Kubicki, M., Acta Crystallographica, Section E., 2012, E68, 01686. doi:10.1107/S1600536812020090.

9. Al, R. K. A.; Abdel, A. H. A., Molecules., 2010, 15, 3775-3815.

10. Borhani, D. W.; Calderwood, D. J.; Frank, K. E. H.; Davis, M.; Josephsohn, N. S.; Skinner, B. S., WO Pat. 2008/063287., 2008.

11. Fidanze, S. D.; Erickson, S. A.; Wang, G. T.; Mantei, R.; Clark, R. F.; Sorensen, B. K.; Bamaung, N. Y.; Kovar, P.; Johnson, E. F.; Swinger, K. K.; Stewart, K. D.; Zhang, Q.; Tucker, L. A.; Pappano, W. N.; Wilsbacher, J. L.; Wang, J., Sheppard, G. S.; Bell, R. L.; Davidsen, S. K.; Hubbard, R. D., Bioorg. Med. Chem. Lett., 2010, 20, 2452-2455.
12. Emmitte, K. A.; Wilson, B. J.; Baum, E. W.; Emerson, H. K.; Kuntz, K. W.; Nailor, K. E.; Salovich, J. M.; Smith, S. C.; Cheung, M.; Gerding, R. M.; Stevens, K. L.; Uehling, D. E.; Jr. Mook, R. A.; Moorthy, G. S.; Dickerson, S. H.; Hassell, A. M.; Leesnitzer, M. A.; Shewchuk, L. M.; Groy, A.; Rowand, J. L.; Anderson, K.; Atkins, C. L.; Yang, J.; Sabbatini, P.; Kumar, R., Bioorg. Med. Chem. Lett., 2009, 19, 1004-1007.

13. Andreani, A.; Burnelli, S.; Granaiola, M.; Guardigli, M.; Leoni, A.; Locatelli, A.; Morigi, R.; Rambaldi, M.; Rizzoli, M.; Varoli, L.; Roda, A., Eur. J. Med. Chem., 2008, 43, 657-661.

14. Andreani, A.; Rambaldi, M.; Andreani, F.; Bossa, R.; Galatulas, I., Eur. J. Med. Chem., 1988, 23, 385-389.

15. Andreani, A.; Rambaldi, M.; Locatelli, A.; Bossa, R.; Fraccari, A.; Galatulas, I., Pharm. Acta Helv., 1993, 68, 21-24.

16. Andreani, A.; Bonazzi, D.; Rambaldi, M., Arch. Pharm., 1982, 315, 451-456.

17. Andreani, A.; Rambaldi, M. M.; Locatelli, A.; Bossa, R.; Fraccari, A.; Galatulas, I., J. Med. Chem., 1992, 35, 4634-4637.

18. Ding, H.; Chen, Z.; Zhang, C.; Xin, T.; Wang, Y.; Song, H.; Jiang, Y.; Chen, Y.; Xu, Y.; Tan, C., Molecules., 2012, 17, 4703-4716.

19. Abdelwareth, S. A. O.; Al, D. A.; Al, M. M. A.; Adra, C. N.; Aboul, F. T., Eur. J. Med. Chem., 2010, 45, 2689-2694.

20. Poorrajab, F.; Ardestani, S. K.; Emami, S.; Behrouzi F. M.; Shafiee, A.; Foroumadi, A., Eur. J. Med. Chem., 2009, 44, 1758-1762.

21. Khalaj, A.; Nakhjiri, M.; Negahbani, A. S.; Samadizadeh, M.; Firoozpour, L.; Rajabalian, S.; Samadi, N.; Faramarzi, M. A.; Adipour, N.; Shafiee, A.; Foroumadi, A., Eur. J. Med. Chem., 2011, 46, 65-70.

22. Vu, C. B.; Bemis, J. E.; Disch, J. S.; Ng, P. Y.; Nunes, J. J.; Milne, J. C.; Carney, D. P.; Lynch, A. V.; Smith, J. J.; Lavu, S.; Lambert, P. D.; Gagne, D. J.; Jirousek, M. R.; Schenk, S.; Olefsky, J. M.; Perni, R. B., J. Med. Chem., 2009, 52, 1275-1283. 
23. Andreani, A.; Rambaldi, M.; Mascellani, G.; Rugarli, P., Eur. J. Med. Chem., 1987, 22, 19-22.

24. Amarouch, H.; Loiseau, P. R.; Bacha, C.; Caujolle, R.; Payard, M.; Loiseau, P. M.; Bories, C.; Gayral, P., Eur. J. Med. Chem., 1987, 22, 463-466.

25. Gupta, G. D.; Jain, K. K.; Gupta, R. P.; Pujari, H. K.; Indian J. Chem., Sect. B.., Org. Chem. Incl. Med. Chem., 1983, 22, 268.

26. Kumaresan, K. L.; Lu, S.; Wen, Y.S.; Hwu, J. R., Organometallics, 1994, 13, 3170-3176.

27. Nagai, K.; Carter, B. J.; Xu, J.; Hecht, S. M., J. Am. Chem. Soc., 1991, 113, 5099-5100.

28. Lobana, T. S.; Bhatia, P. K., J. Sci. Ind. Res., 1989, 48, 394-401.

29. Vogel, A. I., A text book of Quantitative Inorganic Analysis; John Wiley and Sons: New York, (Standard methods)., 1963.

30. Jolley, J.; Cross, W. I.; Pritchard, R. G.; McAuliffe, C. A.; Nolan, K. B., Inorganica Chimica Acta., 2001, 315, 36-43.

31. Kahn, E. S.; Rheingold, A. L.; Shupack, S. I., J. Crystallographic and Spectroscopic Research., 1993, 23(9), 697-710.
32. Trzhtsinskaya, B. V.; Abramova, N. D., Sulphur Reports., 1991, 10(4), 389-430.

33. Liang Y. Q.; Zhao W. Y.; XU W. Q., Acta Chimica Sinica., 1986, 1126, 42-47.

34. Barraclough, C. G.; Kew, D. J., Australian J. Chem., 1970, 23, 2387-2396.

35. Ward, B. G.; Stafford, F. E., Inorg. Chem., 1968, 7, 2569.

36. Bodo, H. H.; Regina., Z. Chem., 1976, 16, 407.

37. Abramenko, V. L.; Sergienko, V. S., Russian J. Inorg. Chem., 2009, 54(13), 2031-2053.

38. Abramenko, V. L.; Sergienko, V.S.; Churakov, A. V., Russian J. Coord. Chem., 2000, 26(12), 866-871.

39. Karpov, M. V.; Davidovich, P. B.; Orlova, D. D.; Belyaev, A. N.; Garabadzhiu, A. V., Russian Journal of General Chemistry., 2015, 85(1), 206-207.

40. http://www.molbase.com/en/hnmr_6857-347-moldata-838140.html\#tabs.

41. http://www.molbase.com/en/name-2Thiazolidinethione.html.

42. http://www.sisweb.com/referenc/tools/ exactmass.htm. 